\title{
Initial Maintenance of Attention to Threat in Children with Social Anxiety Disorder? Findings from an Eye-Tracking Experiment
}

\author{
Steffen Schmidtendorf ${ }^{1}$ (I) - Arvid Herwig ${ }^{2}$. Susanne Wiedau ${ }^{1} \cdot$ Julia Asbrand $^{3} \cdot$ Brunna Tuschen-Caffier $^{3}$. \\ Nina Heinrichs ${ }^{2}$
}

Accepted: 31 May 2021 / Published online: 29 June 2021

(c) The Author(s) 2021

\begin{abstract}
Background Attentional biases are assumed to be a core feature in the etiology and maintenance of clinical anxiety. The present study focuses on initial maintenance of attention to threat, one of three attentional components investigated the least, particularly in child anxiety.

Methods Angry and neutral facial expressions were presented in a free-viewing task, while eye-movements were recorded. Participants were $N=96$ school-aged children, with $n=50$ children with a clinical social anxiety disorder (SAD) and $n=46$ healthy control children (HC). Prior to the task, social stress was induced in half of participating children to investigate the impact of increased levels of distress on initial attention allocation.

Results The length of first fixation to angry faces in children with SAD neither differed from the length of first fixation to neutral faces nor the length of first fixation to angry faces in HC children. Furthermore, this variable was not affected by a stress induction procedure. However, children with SAD initially fixated longer on faces than HC children.

Conclusion Our findings provide evidence for difficulties disengaging attention from faces. This may indicate that attention allocation is determined by the social nature of the stimuli rather than by the specific emotional valence.
\end{abstract}

Keywords Attentional bias $\cdot$ Maintained attention $\cdot$ Pediatric social anxiety $\cdot$ Eye-tracking

Abnormal cognitive processes, including attention to threatrelevant information, are highly relevant in theories on the etiology and maintenance of anxiety disorders in general (e.g., Beck \& Clark, 1997; Mogg \& Bradley, 1998) and social anxiety disorder (SAD) in particular (Clark \& Wells, 1995). There are three different components of biased attention to threat (Cisler \& Koster, 2010), that are discussed in the frame of basically two different hypotheses (Weierich et al., 2008). The vigilance-avoidance hypothesis includes initial hypervigilance (component 1) followed by attentional avoidance (component 2) of threat (Mogg \& Bradley, 1998). Anxious individuals are supposed to initially engage

Steffen Schmidtendorf

s.schmidtendorf@tu-braunschweig.de

1 Department of Psychology, University of Braunschweig, Humboldtstr. 33, 38106 Braunschweig, Germany

2 Department of Psychology, University of Bremen, Bremen, Germany

3 Institute of Psychology, Albert-Ludwigs University of Freiburg, Freiburg, Germany attention more frequently with threatening than with neutral stimuli. Subsequently, they show an increased avoidance of threatening stimuli by disengaging attention away from threat. In contrast, the maintenance hypothesis (Fox et al., 2001) posits maintained attention (component 3 ) to threat, due to difficulty in disengaging attention from threat in anxious individuals. Although avoidance and maintenance seem contradictory on first glance, Weierich et al. (2008) reviewed these hypotheses and suggested an integrated model of attentional processing in anxiety: Initially, anxious individuals direct attention more efficiently to threatening stimuli and once detected, they have more difficulties to disengage from those stimuli. Across time, they avoid threat by inhibiting the return of gaze to the threatening stimuli. It is currently unclear if initial maintenance is a process-oriented component that requires initial hypervigilance as a precursor or whether it is an independent process that may also occur when non-threatening stimuli were fixated first.

While a large body of research gives evidence for an initial hypervigilance (component 1) to threat in adult anxiety (e.g., Armstrong \& Olatunjii, 2012; Bar-Haim et al., 2007), 
the role of initial maintenance of attentional to-or avoidance of-threat (components 2 and 3) remains unclear. Previous research widely investigated biased attention in anxiety with the dot-probe paradigm, which, however, does not allow differentiating definitely between the three components of attentional bias (e.g., Armstrong \& Olatunji, 2012; Clarke et al., 2013). Shorter latencies to identify probes, which are supposed to indicate biased attention to threat, may be determined by selective engagement with threatening stimuli. However, faster reactions may also be explained by an increased tendency to remain in the locus of threatening stimuli. Eye-tracking research enables the dynamic assessment of attention allocation and therefore the separate investigation of initial hypervigilance (component 1) and initial maintenance of attention (component 3 ) to threat.

While the probability to first fixate on a threatening stimulus is typically used to analyze initial hypervigilance, the length of first fixation to a threatening stimulus usually indicates initial maintenance of attention. Eye-tracking experiments in psychological basic research on visual search and reading furthermore assess initial maintenance of attention by analyzing first run dwell time (e.g., Horstmann et al., 2016; Liversedge et al., 1998). In contrast to the length of first fixation, subsequent fixations within an area of interest are included here (beside the first fixation), assuming that individuals maintain attention on a stimulus by fixating on further details. To our knowledge, first run dwell time was not analyzed in clinical research yet. In their metaanalysis, Armstrong and Olatunji (2012) reported inconsistent findings regarding initial maintenance of attention in adult research. Results were moderated by experimental paradigm. A longer first fixation to threat in anxiety was found in visual search tasks, but not in free viewing tasks, which were mostly used. More recent research investigating social anxiety disorder (SAD) in adults revealed further inconsistencies from the eye-tracking free viewing task. While Lazarov et al. (2016) did not find differences in initial maintenance of attention between high, low and clinical levels of social anxiety, Liang et al. (2017) reported initially maintained attention to angry faces in a non-clinical sample in high and low social anxiety. Thus, the link between the third component of attentional bias and anxiety, in particular social anxiety remains unclear. Further, research including patients with diagnosed SAD is necessary to further examine clinical levels of anxiety.

Research investigating biased attention in child anxiety was systematically reviewed in a meta-analysis (Dudeney et al., 2015). The authors reported biased attention to threat in both, anxious and healthy control children. This bias to threat was significantly greater in children with anxiety, relative to healthy control children. However, the effect was smaller and less robust than in adults. Furthermore, age was identified as a significant moderator with a stronger bias between anxious and control children in older than in younger participants (age range 4 to 18 years). Child development may play an important role in attention allocation and therefore contribute distinctly to the reported different outcomes. Field and Lester (2010) proposed a theoretical developmental framework, considering how child development may influence information processing biases, including attentional biases to threat. Previous findings providing evidence for initial hypervigilance in anxious and healthy control children (e.g., Dudeney et al., 2015) are in line with the 'moderation model' of Field and Lester (2010), that concludes an increased shift of attention to threatening stimuli as normative in young children. While non-anxious children may learn to inhibit processing priorities of threatening stimuli, anxious children may not (see Mathews \& Mackintosh, 1998). Other components of biased attention may develop in other ways. According to the 'acquisition model' biases could develop later in adolescence as a function of a child's development and anxiety level. Furthermore, the 'integral bias model' assumes processing biases to be mostly independent from developmental factors, but determined entirely by individual factors (Field \& Lester, 2010). Basic research has also examined the influence of age on eye movements and found differences in face processing between children and adults (e.g., Schwarzer et al., 2005). In a developmental study, Schwarzer et al. (2005) compared eye movements of children aged 6-7 years and 9-10 years with those of adults aged 21-39 years. Photographs of faces were presented and participants were instructed to assign the images to one of two categories. Overall dwell time on faces, as well as the number of fixations, decreased significantly with age.

In line with research on adult anxiety, the most commonly employed paradigms in child anxiety research were static reaction-time measurements. Eye-tracking research on biased attention in anxious children is scarce and mostly focuses on initial hypervigilance or attentional avoidance (e.g., In-Albon et al., 2010; Seefeldt et al., 2014). We recently analyzed both components in a sample of 79 schoolaged children, 37 of them diagnosed with SAD (Schmidtendorf et al., 2018). A hypervigilance-avoidance pattern to angry faces emerged, when they were paired with a nonsocial stimulus. Although children with SAD more often initially directed their gaze to angry faces than did healthy control children, bias scores were below 0.50. As a bias score of 0.50 indicates equal attention distribution across both presented stimuli, these results indicated rather a lack of inhibiting threat representations than a hypervigilance to threat in children with SAD. In our previous work, initial maintenance on threat stimuli has not yet been analyzed, as it requires specific eye-tracking parameters, which had not been extracted for the previous analyses.

To our knowledge, only two recent studies investigated initial maintenance of attention in anxious children 
(Dodd et al., 2015; Pergamin-Hight et al., 2016a). Similar to adult anxiety research, findings are inconsistent with contradictory evidence. Dodd et al. (2015) analyzed the length of first fixation using an eye-tracking free-viewing paradigm (sample: 83 children aged three and four years), which were recorded while picture pairs of angry and neutral faces were presented. The analysis included both differences in the length of first fixation between angry and neutral faces in anxious children (within-subject bias) and differences between anxious children and healthy control children regarding this bias (between-subject bias). In anxious children, the average length of first fixation on angry faces $\left(M_{\text {angry }}=361.21 \mathrm{~ms}, S D_{\text {angry }}=61.08 \mathrm{~ms}\right)$ did not differ significantly from that on neutral faces $\left(M_{\text {neutral }}=356.37 \mathrm{~ms}, S D_{\text {neutral }}=93.73 \mathrm{~ms}\right)$. However, anxious children $\left(M_{A n x}=356.16 \mathrm{~ms}, S D_{A n x}=66 \mathrm{~ms}\right)$ showed shorter first fixations to faces than healthy control children $\left(M_{H C}=398.65 \mathrm{~ms}, S D_{H C}=75.21 \mathrm{~ms}\right)$. Those findings did not support the assumption of initial maintained attention in anxious kids but rather suggested avoidance of face stimuli in general, relative to non-anxious participants. The authors suggested that anxious children interpreted the neutral facial expressions also as threatening and subsequently avoided both stimuli (Dodd et al., 2015). Pergamin-Hight et al. (2016a) employed an emotion spatial-cuing task in 113 youth at the age of 6 to 18 years of whom 71 were diagnosed with a clinical SAD. A target stimulus, which required the participant to press a corresponding button, was presented on the left or right side of the screen, after an angry, neutral or happy face was previously presented on either position. In line with the assumption of initial maintenance of attention to threat, participants with SAD had greater difficulty in disengaging attention from angry faces compared to nonanxious participants. Furthermore, in contrast to initial orienting of attention to angry faces (initial hypervigilance), initial maintenance of attention to angry faces significantly predicted SAD diagnosis in a multivariate model.

The present short report aims to consider initial maintenance of attention (component 3 ) in the same sample of children with SAD above and beyond the already analyzed first and second component of biased attention (Schmidtendorf et al., 2018; see above). Therefore, the present data are taken from a larger research project to investigate cognitive and psychophysiological reactions in children with SAD to emotional stimuli (data collection from 2011 to 2014). In the present analyses, we focus on both, the length of first fixation and first run dwell time in angry-neutral face pairs, a stimulus condition, which was also investigated by Dodd et al. (2015) previously. To extend previous findings on biased attention to threat in child anxiety, we implemented a stress induction condition in half of the participating children. The stress induction procedure that was adapted from Garner et al. (2006), aimed to activate social anxiety schemata according to theoretical assumptions by Clark and Wells (1995). Negative social self-schemata are assumed to increase interpretations of threat in social situations in SAD. Thus, an increased bias could occur in the stress induction condition, specifically in anxious individuals. The probability to first fixate on threatening stimuli has been investigated under stress induction condition in both adult (e.g., Garner et al., 2006) and child (e.g., Seefeldt et al., 2014) anxiety. While Garner et al. (2006) found high socially anxious adults to show a reduced bias in initial orienting to faces, Seefeldt et al. (2014) reported an increase of initial vigilance in children with SAD under social-evaluative stress. To our knowledge, the length of first fixation to threatening stimuli has only been analyzed in the context of a social stressor in adults (Garner et al., 2006). In the stress induction condition, initial maintenance of gaze on faces was reduced in high socially anxious participants.

Based on the theoretical assumption of initial maintenance of attention to threat and findings from previous experiments investigating social anxiety in adolescents with a different experimental task (Pergamin-Hight et al., 2016a) and in adults with the same approach (using eye-tracking, Liang et al., 2017), the following hypotheses were evaluated: (1) The length of first fixation and first run dwell time in children with SAD should be longer to angry faces than to neutral faces, indicating initial maintenance of attention to threat (within-subject bias). (2) Children with SAD should differ from $\mathrm{HC}$ children regarding this bias. Initial maintenance of attention to threat was expected to be greater in children with SAD than in HC children (between-subject bias). (3) This bias is supposed to be affected by a stress induction procedure. In contrast to the first and the second hypothesis, the third one is non-directional, given that the theoretical background does not permit a well-grounded prediction and previous results are lacking.

\section{Method}

\section{Participants}

Initial participants were 122 children (9-13 yrs.), who were recruited for a two-site research project to investigate cognitive and psychophysiological processes in children with SAD. As the data are being used in a large project, this Method section has been reported partly before in a similar fashion (Schmidtendorf et al., 2018). Children assigned to the SAD group met DSM-5 or ICD-10 criteria for a current principal diagnosis of SAD and children in the control group did not meet diagnostic criteria for any current or lifetime diagnosis. After a short screening procedure by phone, trained interviewers with clinical experience assessed clinical status of eligible children with a structured interview 
(Schneider et al., 2009) based on the Anxiety Disorders Interview Schedule for children. To ensure good data quality, we previously applied a very strict algorithm, so that only children with very good data samples were included. This benefit, however, was traded on the cost of a large loss of participants. To improve this cost-benefit ratio, and increase power to detect interaction effects, we sought advice from an expert in experimental psychology and eye movements to review and revise the data exclusion algorithm. The improved algorithm included the following three conditions: (a) the task was successfully completed, (b) the tracking ratio measured at least $80 \%$, and (c) participants made a fixation to at least either picture on a least $75 \%$ of trials. Compared to our previous analyses (Schmidtendorf et al., 2018), the present sample size increased by $n=17$ participants while $N=26$ participants $(n=17 \mathrm{SAD} ; n=9 \mathrm{HC})$ were still excluded from eye movement analysis. Excluded children neither differed significantly in group $(p=0.27)$, age $(p=0.99)$, sex $(p=0.82)$, stress induction condition $(p=0.38)$ or study site $(p=0.12)$ nor in self-reported symptom severity $(p=0.22)$ or number of comorbid diagnoses in SAD (all $p=0.17$ ). The final sample for the eye-tracking task ( $n=96 ; 79 \%)$ consisted of $n=50$ children with a primary SAD (with comorbidity allowed) and $n=46$ healthy control children.

Referring to previous findings, concretely a medium effect in Pergamin-Hight et al. (2016a; $d=0.4)$ and a large effect in Liang et al. $(2017 ; d=0.7)$, our sample size $(N=96)$ provided a power of $1-\beta=0.62-0.96$ to uncover a significant difference between groups in the length of first fixation/ first run dwell time to angry faces.

\section{Measures}

Social anxiety symptoms were assessed using the Social Phobia and Anxiety Inventory for Children (SPAI-C; Beidel et al., 1995; Melfsen et al., 2001) and the Social Anxiety Scale for Children Revised (SASC-R; La Greca \& Stone, 1993; Melfsen \& Florin, 1997). Likert scaling consists of three-points in the SPAI-C $(0=$ never or hardly ever to $2=$ almost always or always), and five points in the SASC$\mathrm{R}(1=$ not at all to $5=$ all the time). In the present sample, Cronbach's Alpha was $a>0.95$ for both instruments.

\section{Materials and Apparatus}

The additional analyses presented here, aim to clarify previous findings on initial maintenance in child anxiety, before extending and exploring further information processing contexts. Given that previous findings (Dodd et al., 2015) are limited to angry-neutral face pairs, the present short report will also focus on the angry-neutral stimulus condition. The entire stimulus set, employed in this experiment, consisted of 30 pairs of pictures. Angry facial expressions were combined with neutral and happy expressions and pictures of houses ( $n=10$, respectively). $N=10$ actors presented angry, happy and neutral expressions, respectively and were balanced regarding age (adults vs. children) and gender (male vs. female). All stimuli were colored, measured approximately $12.8 \mathrm{~cm} \times 9.1 \mathrm{~cm}\left(12.1^{\circ} \times 8.7^{\circ}\right)$ and were matched in complexity. Our previous analyses of the recorded eye tracking data (Schmidtendorf et al., 2018) were based on areas of interest (AoI) that were closely drawn on the presented faces and houses. To consider the size of the fovea and possible imprecisions of the eye-tracker (see Holmqvist et al., 2011, p. 223), the size of the AoI were adapted to the facial stimuli used for the present analyses. Any AoI on each stimulus was therefore now circular and of identical size, with a diameter of approximately $16.1 \mathrm{~cm}\left(15^{\circ}\right)$. They were arranged cattycornered with a horizontal distance of $6.1 \mathrm{~cm}\left(5.8^{\circ}\right)$ and a vertical distance of $9.3 \mathrm{~cm}\left(8.8^{\circ}\right)$ from the center. The position of each stimulus was counterbalanced regarding its valence (negative, neutral, positive and non-social) as well as regarding age and gender of the person pictured. A central fixation cross, measuring about $5 \mathrm{~cm} \times 5 \mathrm{~cm}$, was presented between trials. The background color for all stimuli and the fixation cross was gray. Children sat on a chair approximately $60 \mathrm{~cm}$ away from a 17"-monitor with a pixel resolution of $1024 \times 768$. The entire task comprised of two blocks of 30 trials, the order of trials was randomized within each block. The stimuli were presented via Presentation Version 12.1 software (Neurobehavioral Systems, Albany, California). Eye movements were recorded using a tower-mounted iView Eye Tracker (SensoMotoric Instruments GmbH, Germany) with a ratio of 1250 or $240 \mathrm{~Hz}$. Overall lengths of first fixation from both sites were similar $\left(t_{94}=0.61, p=0.55\right)$, thus the different sampling frequency did not impact upon lengths.

\section{Procedure}

Prior to the first assessment, all children and parents completed a consent form, which was approved by an ethical review board. Children received a voucher (amount: 25 Euro), and parents received an additional 35 Euro in cash for project participation. At the beginning of the task, children were told that their eye movements would be recorded, while being presented with a fixation cross and pictures of faces and houses. However, they were not instructed to fixate on the presented stimuli. They were instructed to look at the central fixation cross when it was visible on the screen. Therefore, only passive viewing was demanded for this task. Prior to the implementation of the stress induction condition, we assessed children's present anxiety on a 0-10 Likert scale. Children who were randomly assigned to the stress induction condition ( $n=23 \mathrm{SAD}$ and $n=22 \mathrm{HC}$ ) were told 
that they would give a short presentation in front of a video camera after the visual attention task. To prepare for the presentation, the participants would receive a short text and be given five minutes to prepare. Their performance of the presentation would then later be evaluated through their video recording. To check the success and extent of this anxiety induction, children in the stress induction condition repeatedly rated their anxiety. The experiment then started with the presentation of four probe trials, followed by a standard 5-point calibration procedure. Prior to each trial, a fixation cross was presented for a variable duration between 750 and $1250 \mathrm{~ms}$. The inter-trial interval varied randomly to prevent predictability of trial onset and reduce the monotony of the task. Each picture pair was presented for $5000 \mathrm{~ms}$, yielding a total task duration of approximately six minutes.

\section{Preparation and Analysis of Data}

Data were prepared and analyzed with BeGaze3 Software (Sensomotoric Instruments GmbH, Germany). As discussed earlier, theoretical assumptions propose initial hypervigilance and initial maintenance as discrete components of biased attention to threat. Likewise, basic research on active vision revealed that during a single fixation foveal analysis occurs independently and in parallel to peripheral target selection (Ludwig et al., 2014). In contrast to previous studies (Dodd et al., 2015; Liang et al., 2017), initial maintenance of attention (as a process based on foveal analysis) thus was calculated irrespective of initial hypervigilance (as a process based on peripheral target selection). We calculated the average length of first fixation to angry faces by dividing the sum of all first fixation durations to angry faces by the number of trials in which the angry face was fixated. The average length of first fixation to neutral faces was calculated similarly. In addition to the average length of first fixation, we further analyzed the average first run dwell time as an indicator for initial maintenance of attention. The average first run dwell time to angry and accordingly neutral faces was taken as the sum of the duration of the first fixation to the corresponding face and all subsequent fixations before the eyes begin to leave the face (Horstmann et al., 2016). Following previous studies (e.g., Dodd et al., 2015; Garner et al., 2006), fixations were counted if they hit one of the two presented faces and lasted for $100 \mathrm{~ms}$ or longer.

Length of first fixation and first run dwell time was analyzed within children with SAD using Wilcoxon signed-rank test and between children with SAD and HC children using a three-way mixed ANCOVA. Age was used as the covariate in the analyses to control for developmental differences even within the narrow age range examined. All statistical analyses were performed with SPSS 26 (SPSS Inc., USA) with an alpha level of 0.05 .
To contextualize present findings on initial maintenance of attention with the results from the other two components, we provide a detailed electronic supplement with further analyses. We also summarize the main outcomes of these further analyses at the end of the result section. The electronic supplement includes (1) Results of initial maintenance of attention as a function of initial hypervigilance (i.e., length of first fixation), to allow comparability with previous findings; (2) The proportion and the latency of first fixation is moreover analyzed to investigate initial hypervigilance in the present sample; (3) Results relating to biased attention in the time course, overall dwell time to angry and neutral faces as well as to the blank area. The blank area was added to the analysis, given that Dodd et al. (2015) found a significant difference in overall dwell time to the blank area between anxious and non-anxious children with a higher value in the latter group. And (4) We analyze the association between measures of maintained attention and self-reported symptom severity to include a dimensional perspective of social anxiety.

\section{Results}

\section{Descriptive Statistics}

Descriptive statistics of participating children are presented in Table 1. Both groups did not differ regarding age $(d=0.07)$ and sex $(d=0.05)$. As expected, self-reported symptom severity was significantly higher in children with SAD in the SPAI-C $(d=2.54)$ and in both subscales of the SASC-R (fear of negative evaluation: $d=1.30$; social avoidance and distress: $d=2.51$ ).

\section{Preceding Results: Effect of Stress Induction on Self-Reported Anxiety}

Anxiety ratings of children who were assigned to the stress induction condition were entered into a two-way mixed ANOVA with group (SAD and HC) as between-subjects factor and time (prior to and directly after stress induction) as within-subjects factor. No significant interaction emerged, $F(1,42)=2.31, p=0.14, \eta_{\mathrm{p}}^{2}=0.052$. However, there was a significant main effect of time $(F(1,42)=6.886$, $\left.p=0.012, \eta_{\mathrm{p}}{ }^{2}=0.141\right)$, demonstrating an increase of anxiety after the stress indcuction procedure in all children $\left(M_{\text {pre }}=0.89, S D_{\text {pre }}=1.24, M_{\text {post }}=1.32, S D_{\text {post }}=1.89\right)$ and a significant main effect of $\operatorname{group}(F(1,42)=6.891, p=0.012$, $\left.\eta_{\mathrm{p}}{ }^{2}=0.141\right)$ with higher anxiety ratings in children with SAD than in HC children $\left(M_{S A D}=1.16, S D_{S A D}=0.73\right.$, $M_{H C}=0.73, S D_{H C}=0.40$ ) before and after the instruction. 
Table 1 Demographics and questionnaire measures as a function of diagnostic group

\begin{tabular}{|c|c|c|c|c|}
\hline & Children with SAD $(n=50)$ & HC children $(n=46)$ & Test value & $p$ \\
\hline \multicolumn{5}{|l|}{ Demographics } \\
\hline Age (years) & $11.4(1.3)$ & $11.5(1.4)$ & $Z=-0.40$ & .689 \\
\hline Sex ( $\%$ female $)$ & 58 & 63 & $\chi^{2}=.26$ & .614 \\
\hline SPAI-C (raw score) & $22.2(9.9)$ & $2.9(3.6)$ & $t_{91}=-12.72$ & $<.001$ \\
\hline Raw score, separated by sex & Boys: 17.2 (7.8) | Girls: 25.7 (9.8) & Boys: 3.2 (4.7) | Girls: 2.7 (2.8) & & \\
\hline T-score range, separated by sex & Boys: $58-63$ । Girls: $58-63$ & Boys: 35 - 37 | Girls: 28 - 34 & & \\
\hline SASC-R: FNE (raw score) & $23.7(8.9)$ & $14.3(4.8)$ & $Z=-5.71$ & $<.001$ \\
\hline Raw score, separated by sex & Boys: 19.6 (6.2) | Girls: 26.8 (9.4) & Boys: 13.6 (4.4) | Girls: 14.7 (5.0) & & \\
\hline T-score range, separated by sex & Boys: $50-57$ I Girls: $58-63$ & Boys: $38-43$ | Girls: $35-37$ & & \\
\hline SASC-R: SAD (raw score) & $26.0(6.6)$ & $12.4(3.8)$ & $Z=-7.80$ & $<.001$ \\
\hline Raw score, separated by sex & Boys: 22.3 (5.3) | Girls: 28.7 (6.3) & Boys: 12.8 (3.1) | Girls: 12.2 (4.2) & & \\
\hline T-score range, separated by sex & Boys: $58-63$ । Girls: $64-67$ & Boys: $35-37$ | Girls: $38-43$ & & \\
\hline
\end{tabular}

$S P A I-C$ Social phobia and anxiety inventory for children $(0-52)$, SASC-R Social anxiety scale for children revised (18 - 90), FNE Fear of negative evaluation, $S A D$ Social avoidance and distress; Standard deviations in parentheses

\section{Initial Maintenance of Attention to Threat within Children with SAD}

The length of first fixation was examined with Wilcoxon signed-rank test. There was no significant difference between the length of first fixation to angry faces and neutral faces in children with SAD, $Z=-0.35, p=0.73, d=0.10$.

Wilcoxon signed-rank test furthermore yielded no significant difference between first run dwell time to angry faces and neutral faces, $Z=-1.36, p=0.18, d=0.39$.

\section{Initial Maintenance of Attention to Threat between Children with SAD and HC Children}

A three-way mixed ANCOVA with group (SAD and HC) and stress induction condition (SI and noSI) as betweensubjects factors and facial expression (angry and neutral) as within-subjects factor was conducted, to examine if groups differ in the length of first fixation and if the length of first fixation is affected by a stress induction procedure. Age was used as the covariate. The interaction effect between group and facial expression was not statistical significant $(F(1$, $\left.91)=0.06, p=0.80, \eta_{\mathrm{p}}{ }^{2}=0.001\right)$. Furthermore, the threeway interaction between group, facial expression and stress induction condition was non-significant $(F(1,91)=3.36$, $p=0.07, \eta_{\mathrm{p}}{ }^{2}=0.036$ ). However, we found a significant main effect for group $\left(F(1,91)=4.59, p=0.04, \eta_{\mathrm{p}}{ }^{2}=0.048\right)$. The mean length of first fixation was higher in children with $\operatorname{SAD}(M=268 \mathrm{~ms}, S D=70 \mathrm{~ms})$ than in HC children $(M=241 \mathrm{~ms}, S D=50 \mathrm{~ms})$. All other interaction and main effects were non-significant (all $F(1,91)<1.58$, all $p>0.21$, all $\left.\eta_{\mathrm{p}}^{2}<0.018\right)$. The mean length of first fixation by group, condition and facial expression is displayed in Fig. 1.
The same analysis was additionally performed with the first run dwell time as dependent variable. The ANCOVA revealed a significant interaction between group and stress induction condition $\left(F(1,91)=4.71, p=0.03, \eta_{\mathrm{p}}{ }^{2}=0.049\right)$. Post-hoc tests indicated, that first run dwell time to faces was affected by a stress induction in HC children $(t(44)=2.41$, $p=0.02, d=0.71)$, with shorter first run dwell time in the stress induction condition $\left(M_{n o S I}=1025 \mathrm{~ms}, S D_{\text {noSI }}=398\right.$, $M_{S I}=787 \mathrm{~ms}, S D_{S I}=243 \mathrm{~ms}$ ). Children with SAD did not differ in first run dwell time to faces with/ without prior stress induction procedure $(t(48)=-0.45, p=0.66$, $d=0.13$ ). Furthermore, we found a significant relationship between the covariate and first run dwell time $(F(1$, $\left.91)=8.97, p<0.01, \eta_{\mathrm{p}}{ }^{2}=0.090\right)$. Post-hoc calculated nonparametric correlation revealed longer first run dwell time to faces in older children than in younger children $(r=0.27$, $p=0.01)$. All other interaction and main effects were nonsignificant (all $F(1,91)<3.46$, all $p>0.06$, all $\eta_{\mathrm{p}}{ }^{2}<0.038$ ). Figure 2 presents first run dwell time for both groups by condition and facial expression. ${ }^{1}$

\footnotetext{
1 As can be seen in Figs. 1 and 2, standard deviations of both eyetracking variables were large in some cells, indicating large inter-individual variability across children within group. In testing if our findings were driven by some extreme values, we repeated the analyses excluding outliers (if length of first fixation and first run dwell time respectively fell more than $2.5 S D$ away from the mean). Hypotheses testing after outlier exclusion revealed similar results for both variables regarding the within-group and the between-group comparison. Although there was another significant main effect for stress induction condition in the ANCOVA analyzing first run dwell time, it is not meaningful, given that it was qualified by a significant interaction and post-hoc tests revealed a significant difference between both conditions only in $\mathrm{HC}$ children. All statistical values of the performed analyses after the exclusion of outliers are presented in table 2 in the electronic supplement.
} 


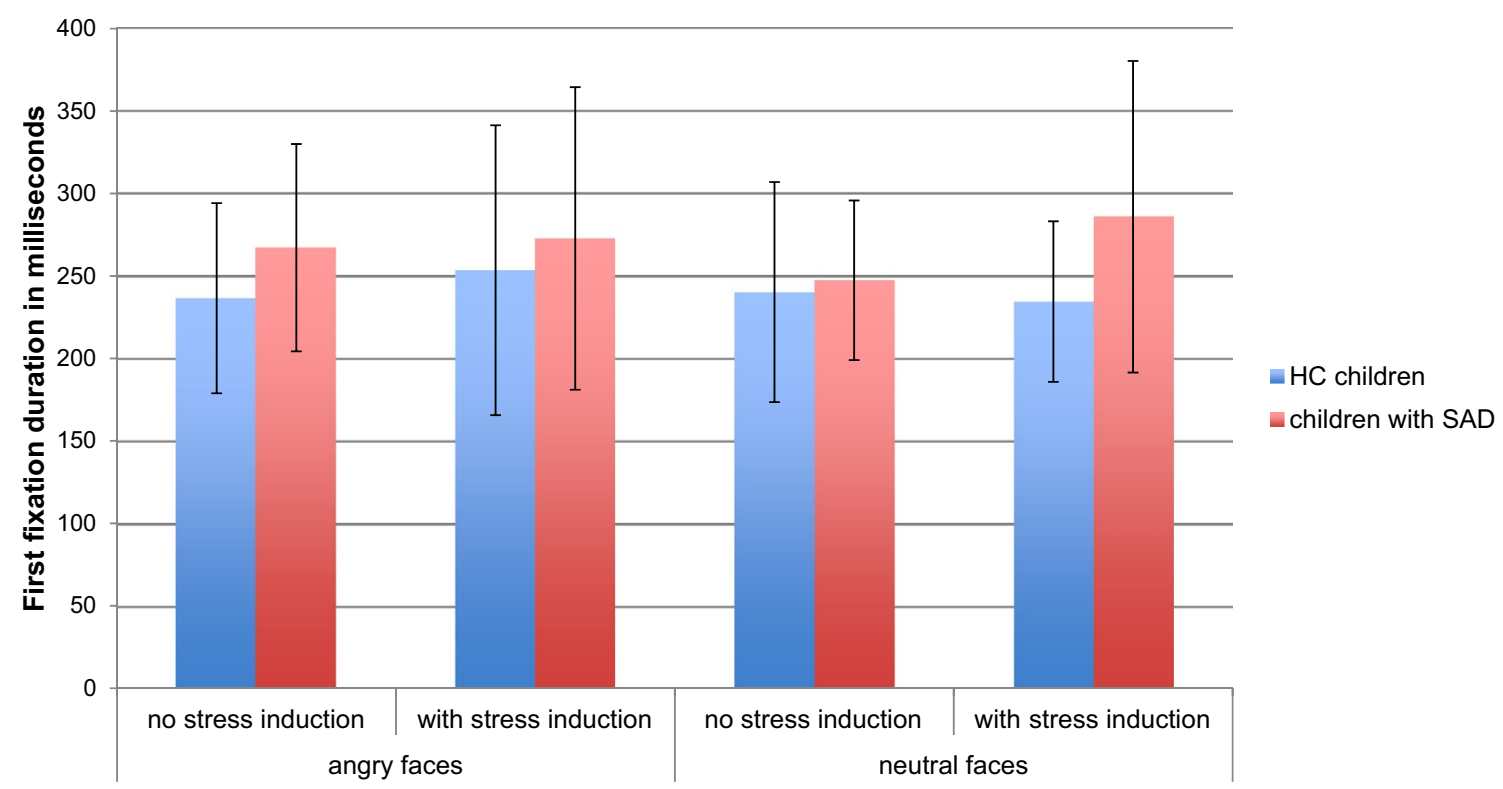

Fig. 1 Length of first fixation in milliseconds for both groups by facial expression and stress induction condition

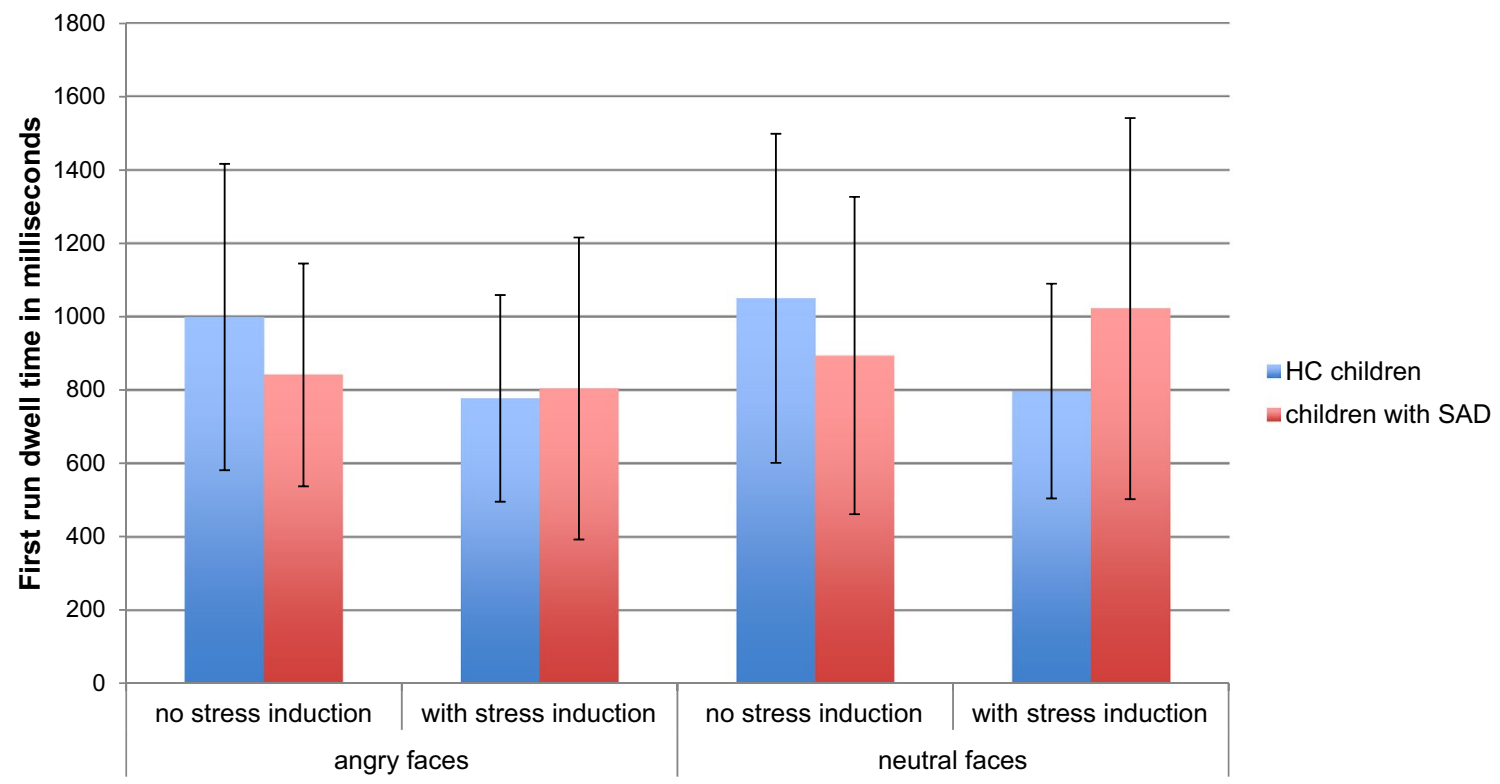

Fig. 2 First run dwell time in milliseconds by group, facial expression and stress induction condition

\section{Contextual Results (See Supplement for Further Information)}

\section{Initial Hypervigilance}

The proportion of first fixation rather proposes initial avoidance of than initial hypervigilance to angry faces in all children. Bias scores are very similar, compared to our previous analysis in a smaller subgroup of the present sample (Schmidtendorf et al., 2018). The probability of first fixation to angry faces did not differ between groups. A stress induction procedure was associated with an increased allocation of attention to angry faces, however, this effect was not statistical significant. Children with SAD and HC children did not differ regarding time to first fixation to angry faces. 


\section{Attentional Avoidance}

The analysis yielded a significant shorter overall dwell time to angry faces and a significant longer overall dwell time to the blank area in children with SAD than in HC children.

\section{Association Between Measures of Maintained Attention and Self-reported Symptom Severity}

Post-hoc calculated Spearman coefficients revealed no significant association between the level of child anxiety and the length of first fixation or first run dwell time to angry faces.

\section{Discussion}

In the present short report, we investigated initial maintenance of attention to threat in children with SAD by analyzing two dependent variables: Length of first fixation and first run dwell time. Half of participating children were assigned to a stress induction condition to activate social self-schemata, which are supposed to affect biased attention to threat. Additionally, we provided further analyses (in the electronic supplement) to place initial maintenance of attention in the context of the other components of biased attention.

\section{Biased Attention to Threat within Children with SAD (within-Group Effects; Hypothesis 1)}

The first hypothesis, predicting longer first fixations and a longer first run dwell time to angry than to neutral faces in children with SAD, was not supported by our data. The results therefore are in line with Dodd et al. (2015), even though they used a much younger sample in their study. The results contradict the assumption of initial maintenance of attention to threat within children with SAD. In line with our previous analyses in a smaller subsample (Schmidtendorf et al., 2018), the contextual analyses with this larger sample also did not yield any indication for initial hypervigilance to angry faces. Children with SAD as well as HC children rather seem to avoid angry faces in favor of neutral faces.

\section{Biased Attention to Threat between Children with SAD and HC Children (between Groups Effects; Hypothesis 2)}

We did not find longer first fixations and longer first run dwell time to angry faces in children with SAD than in HC children. However, length of first fixation to faces was higher in children with SAD than in HC children. Anxious children seem to maintain attention on facial stimuli, irrespective of their valence.
Our finding may be compatible with initial maintenance of attention to threat, when considering interpretive biases in social anxiety (e.g., Yoon \& Zinbarg, 2008). Given the ambiguity of neutral faces, anxious children may initially interpret those faces as threatening as well, resulting in difficulties disengage attention away from both facial stimuli.

It is important to note, that we only found a significant difference between groups, when length of first fixation was examined irrespective of the proportion of first fixation to angry and neutral faces, respectively. To allow comparability with the existing literature, the contextual analyses provide additional analyses of initial hypervigilance and the length of first fixation according to initial hypervigilance. In the present sample, the probability of first fixation to angry faces in favor of neutral faces did not differ between groups. On the one hand, this falls in line with previous results (e.g., Dodd et al., 2015; In-Albon et al., 2010) that also revealed no evidence for initial hypervigilance to threat in anxious children. On the other hand, given the mixed nature of previous results, our findings are in contrast to Shechner et al. (2013) and a recent meta-analysis (Dudeney et al., 2015), indeed reporting more vigilance to threat in anxious compared to non-anxious participants. Consistent with Shechner et al. (2013), time to first fixation to angry faces did not differ between groups. To our knowledge, clinical eye-tracking experiments only assessed initial maintenance of attention on the condition of initial hypervigilance so far (Dodd et al., 2015 in child anxiety; Lazarov et al., 2016 and Liang et al., 2017 in adult SAD). In line with Lazarov et al. (2016), but in contrast to Dodd et al. (2015) and Liang et al. (2017), the length of first fixation presuming initial hypervigilance (i.e., the analysis includes only trials, in which the angry face was initially fixated) did not differ between groups.

First run dwell time, as a broader definition of initial maintenance of attention, includes subsequent fixations within the initially fixated face, before the eyes begin to leave the face. The analyses did not reveal a significant difference between both groups. Our contextual analysis regarding overall dwell time to angry and neutral faces as well as to the blank area revealed a significant difference between groups with respect to angry faces and the blank area. Overall dwell time to angry faces was shorter in children with SAD than in HC children. As suggested by Garner et al. (2006) anxious children may strategically reduce attention to threat. Furthermore, in line with Dodd et al. (2015), overall dwell time to the blank area was longer in children with SAD than in HC children. Compared to HC children, children with SAD may be more likely to fixate on the blank area, as it offers the only occasion to avoid facial stimuli, in particular angry faces. While length of first fixation suggests difficulties in disengaging attention from threat in initial attention allocation in children with SAD, overall dwell time seems compatible with theoretical assumptions of 
attentional avoidance of threat in later stages of information processing (Williams et al., 1988).

\section{Initial Attention Allocation and Social Stress}

We implemented a stress induction procedure to test a selected aspect of a cognitive model of social anxiety (Clark \& Wells, 1995), which assumed negative social self-schemata to increase interpretations of threat in social situations. The third hypothesis, proposing a stress induction procedure affecting initial attention allocation, was supported only for first run dwell time.

While Garner et al. (2006) reported shorter first fixations to faces under social stress in high anxious adults compared to low anxious adults, we failed to find an association between a stress induction and the length of first fixation in the present experiment. Several reasons may be taken into account to explain the differing findings. First, participants may differ regarding the induced anxiety level between studies. Although, the anxiety ratings prior to and after the stress induction procedure indicated an increase in anxiety in our experiment, the self-reported levels of child distress were quite low. Furthermore, the effect of the procedure was medium $\left(\eta_{p}{ }^{2}=0.14\right)$ in the present experiment, while it was large in the experiment conducted by Garner et al. $\left(\eta_{\mathrm{p}}{ }^{2}=0.43\right)$. Second, children in the control condition were asked to rate their levels of anxiety only prior to the stress induction procedure. Therefore, it cannot be excluded that the between-groups effect of the stress induction procedure may have been reduced by an increase in anxiety, even in the control group. Third, Garner et al. included happy-neutral face pairs in their analyses, while our present results are confined to angryneutral face pairs. Fourth, participants were school-aged children in our experiment and undergraduate students in Garner et al. (2006). This seems to be of importance, given that age was recently found to moderate biased attention in anxiety (Dudeney et al., 2015; see discussion below).

The second dependent variable, first run dwell time, was differentially affected by a stress induction procedure. Within the group of $\mathrm{HC}$ children, first run dwell time was shorter in the stress induction condition. Children with SAD did not differ between both conditions. When considering all subsequent fixations as potentially influenced by conscious and strategical processes of attention allocation, our results regarding first run dwell time suggest avoidance of faces when greater levels of stress were experienced. Increasing state anxiety may function as a facilitator to disengage attention from facial stimuli - however, only in healthy participants. Considering that anxious children may initially interpret neutral faces as threatening as well, the present results are therefore partially consistent with the assumption of difficulties disengaging attention from threat in children with SAD, compared to HC children.

\section{General Discussion}

In sum, we failed to find clear evidence for initial maintenance of attention to threat in children, which actually is assumed to be a core feature of biased attention in anxiety (e.g., Fox et al., 2001). Several studies recently investigated this component of biased attention in adult (Lazarov et al., 2016; Liang et al., 2017) and pediatric (Dodd et al., 2015; Pergamin-Hight et al., 2016a) SAD. In line with Dodd et al. (2015), who investigated initial maintenance of attention to threat in very young children, aged three to four years, we neither found indication for biased attention to angry faces within children with SAD nor between children with SAD and HC children. Based on the moderating effect of age on attentional bias between anxious and healthy children (Dudeney et al., 2015) and some recent findings reporting initial maintenance of attention to threat in adults (Liang et al., 2017) and youth with SAD (Pergamin-Hight et al., 2016a; age range $=6-18$ years), the 'acquisition model' may be most appropriate to classify the inconsistent findings (Field \& Lester, 2010). According to this model, initial maintenance of attention to threat may develop later in adolescence as a function of a child's development and anxiety level. The inclusion of age in the analyses revealed a significant association between age and first run dwell time with longer first run dwell time in older children. This finding is not in line with findings from basic research (e.g., Schwarzer et al., 2005), reporting overall dwell time on faces, as well as the number of fixations decreasing significantly with age. However, Schwarzer et al. (2005) did not analyze initial attentional allocation separately. Considering that the present effect of age did not interact with anxiety, stimulus valence, or the stress induction condition in our analyses, it is possible that differences between older and younger children in first run dwell time already occur during the visual encoding phase of face processing and are independent of anxiety and threat. For future research, an interesting approach would be to compare child and adult individuals with social anxiety using the same experiments to investigate the impact of learning processes and developmental factors on attentional biases.

There are some limitations to the present study that need to be acknowledged. As discussed by Armstrong and Olatunji (2012), the free-viewing paradigm may be less suitable to assess initial maintenance of attention to threat than the visual search paradigm, given that participants were not asked explicitly to disengage attention from threat. Another explanation may lie in the required depth of processing of the stimuli in the two paradigms. In visual search tasks, for each fixation on a stimulus, it must be decided whether the 
stimulus is a target or a distractor. This requires matching the stimulus with an internal representation of the target (target template matching). With increasing target-distractor similarity, this process requires an increasingly detailed analysis of the stimulus. Thus, the probability to process the threatpotential of a distractor is increased in visual search tasks, which can prolong the initial attention allocation. In free viewing tasks, however, there is no need for such a detailed analysis of the stimulus; a superficial processing is sufficient. Accordingly, participants do not necessarily need to process the threat-potential of a stimulus. Furthermore, covert attentional processes in initial maintenance of attention, which were not captured by eye-tracking, were taken into consideration to explain, why previous free-viewing studies failed to observe this bias (see also Garner et al., 2006). However, the precise nature of the maintenance bias remains unclear yet and it was recently found in a free-viewing eyetracking experiment in socially anxious adults (Liang et al., 2017). To date, as stated earlier, there is only little research on this component of attentional bias in child anxiety. Our study provides eye-movement data in a homogeneous, carefully assessed clinical sample that was analyzed in the same way as Dodd et al. (2015). The present study is the first to examine initial maintenance of attention to threat not only subsequent to initial hypervigilance, but also dissociated from it. Moreover, we investigated the impact of increased levels of distress on the length of first fixation in pediatric anxiety by performing a stress induction procedure prior to data recording.

For attention bias modification (ABM) techniques, which are increasingly discussed and investigated as interventions for anxiety disorders, the concrete nature of biased attention seems to be crucial, as a reduction of anxiety is to be achieved, explicitly by reducing biased attention. MacLeod and Clarke (2015) reported a reduction in anxiety vulnerability and symptomatology in those interventions that successfully reduced biased attention. However, they also found a substantial proportion of studies failing to reduce attentional selectivity as intended. In their meta-analysis, Heeren et al. (2015) found a significant reduction in SAD symptoms after treatment, but not at 4-month follow-up. The authors noted that ABM techniques need further development before they can be widely used in clinical practice. Moreover, PergaminHight et al. (2016b) who failed to find clear evidence for $\mathrm{ABM}$ relative to a control condition in youth with SAD, emphasized, that developmental influences need to be considered in the implementation of ABM.

Another, at this stage even more promising new potential clinical strategy for SAD is virtual reality exposure therapy (VRET; e.g., Pelissolo et al., 2019). In a recent meta-analysis, Carl et al. (2019) found VRET to be more effective than waitlist and placebo groups. With regard to in vivo exposure therapy, there was no significant difference, leading to the conclusion, that VRET is an equivalent treatment technique to in vivo exposure therapy. To date, in most studies that investigated VRET in comparison to in vivo exposure therapy in SAD, VRET has been combined with cognitive interventions (see Emmelkamp et al., 2020). With the incorporation of current technological developments into VRET, sufficient technical conditions could be created to combine exposure therapy and attention training techniques in one strategy. Considering the promising nature of ABM (see Pelissolo et al., 2019), the combination of both therapeutic strategies may be suitable to increase efficacy of treatment in patients with SAD.

Our present results provide some evidence for biased attention to social stimuli in school-aged children with $\mathrm{SAD}$, in particular difficulties in disengaging attention from faces in early stages of information processing, followed by attentional avoidance of faces. This finding further supports the rationale to develop novel attention training procedures for the treatment of SAD. However, given that this finding was only found, when initial maintenance of attention was analyzed irrespective of initial hypervigilance and is only partially in line with theoretical assumptions and some previous work, prior to the implementation and evaluation of bias modification procedures in clinical pediatric anxiety, more research is needed, to further understand the nature of biased attention in this population.

Supplementary Information The online version contains supplementary material available at https://doi.org/10.1007/s10608-021-10244-2.

Acknowledgements We are grateful for the ananymous reviewers' encouragement to use age as a covariate and include more information on why a developmental perspective may be helpful in attention biases in children in the introduction.

Funding Open Access funding enabled and organized by Projekt DEAL. This research was supported by a Grant from the German Research Foundation (DFG) to Nina Heinrichs (HE 3342/4-2) and Brunna Tuschen-Caffier (TU 78/5-2).

\section{Declarations}

Conflict of Interest This work should be part of the cumulative dissertation of Steffen Schmidtendorf, which is supervised by Nina Heinrichs.

Ethical Approval All procedures performed in studies involving human participants were in accordance with the ethical standards of the institutional and/or national research committee and with the 1964 Helsinki declaration and its later amendments or comparable ethical standards.

Informed Consent Informed consent was obtained from all individual participants included in the study.

Open Access This article is licensed under a Creative Commons Attribution 4.0 International License, which permits use, sharing, adaptation, distribution and reproduction in any medium or format, as long 
as you give appropriate credit to the original author(s) and the source, provide a link to the Creative Commons licence, and indicate if changes were made. The images or other third party material in this article are included in the article's Creative Commons licence, unless indicated otherwise in a credit line to the material. If material is not included in the article's Creative Commons licence and your intended use is not permitted by statutory regulation or exceeds the permitted use, you will need to obtain permission directly from the copyright holder. To view a copy of this licence, visit http://creativecommons.org/licenses/by/4.0/.

\section{References}

Armstrong, T., \& Olatunji, B. O. (2012). Eye tracking of attention in the affective disorders: A meta-analytic review and synthesis. Clinical Psychology Review, 32(8), 704-723. https://doi.org/10. 1016/j.cpr.2012.09.004

Bar-Haim, Y., Lamy, D., Pergamin, L., Bakermans-Kranenburg, M. J., \& van Ijzendoorn, M. H. (2007). Threat-related attentional bias in anxious and nonanxious individuals: A meta-analytic study. Psychological Bulletin, 133(1), 1-24. https://doi.org/10.1037/ 0033-2909.133.1.1

Beck, A. T., \& Clark, D. A. (1997). An information processing model of anxiety: Automatic and strategic processes. Behaviour Research and Therapy, 35(1), 49-58. https://doi.org/10.1016/ S0005-7967(96)00069-1

Beidel, D. C., Turner, S. M., \& Morris, T. L. (1995). A new inventory to assess childhood social anxiety and phobia: The social phobia and anxiety inventory for children. Psychological Assessment, 7(1), 73-79. https://doi.org/10.1037/1040-3590.7.1.73

Carl, E., Stein, A. T., Levihn-Coon, A., Pogue, J. R., Rothbaum, B., Emmelkamp, P., \& Powers, M. B. (2019). Virtual reality exposure therapy for anxiety and related disorders: A meta-analysis of randomized controlled trials. Journal of Anxiety Disorders, 61, 27-36. https://doi.org/10.1016/j.janxdis.2018.08.003

Cisler, J. M., \& Koster, E. H. (2010). Mechanisms of attentional biases towards threat in anxiety disorders: An integrative. Clinical Psychology Review, 30(2), 203-216. https://doi.org/10.1016/j.cpr. 2009.11.003

Clark, D. M., \& Wells, A. (1995). A cognitive model of social phobia. In R. G. Heimberg, M. R. Liebowitz, D. A. Hope \& F. R. Schneider (Eds.), Social phobia: Diagnosis, assessment, and treatment (pp. 69-93). New York: Guilford.

Clarke, P. J., MacLeod, C., \& Guastella, A. J. (2013). Assessing the role of spatial engagement and disengagement of attention in anxiety-linked attentional bias: A critique of current paradigms and suggestions for future research directions. Anxiety, Stress and Coping, 26(1), 1-19. https://doi.org/10.1080/10615806.2011. 638054

Dodd, H. F., Hudson, J. L., Williams, T., Morris, T., Lazarus, R. S., \& Byrow, Y. (2015). Anxiety and attentional bias in preschoolaged children: An eye tracking study. Journal of Abnormal Child Psychology, 43(6), 1055-1065. https://doi.org/10.1007/ s10802-014-9962-x

Dudeney, J., Sharpe, L., \& Hunt, C. (2015). Attentional bias towards threatening stimuli in children with anxiety: A meta-analysis. Clinical Psychology Review, 40, 66-75. https://doi.org/10.1016/j. cpr.2015.05.007

Emmelkamp, P. M. G., Meyerbröker, K., \& Morina, N. (2020). Virtual reality therapy in social anxiety disorder. Current Psychiatry Reports, 22, 32. https://doi.org/10.1007/s11920-020-01156-1

Field, A. P., \& Lester, K. J. (2010). Is there room for 'development' in developmental models of information processing biases to threat in children and adolescents? Clinical Child and Family Psychology Review, 13(4), 315-332.

Fox, E., Russo, R., Bowles, R., \& Dutton, K. (2001). Do threatening stimuli draw or hold visual attention in subclinical anxiety? Journal of Experimental Psychology: General, 130(4), 681-700. https://doi.org/10.1037/0096-3445.130.4.681

Garner, M., Mogg, K., \& Bradley, B. P. (2006). Orienting and maintenance of gaze to facial expressions in social anxiety. Journal of Abnormal Psychology, 115(4), 760-770. https://doi.org/10.1037/ 0021-843X.115.4.760

Heeren, A., Mogoaşe, C., Philippot, P., \& McNally, R. J. (2015). Attention bias modification for social anxiety: A systematic review and meta-analysis. Clinical Psychology Review, 40, 76-90. https://doi. org/10.1016/j.cpr.2015.06.001

Holmqvist, K., Nyström, M., Andersson, R., Dewhurst, R., Jarodzka, H., \& Van de Weijer, J. (2011). Eye tracking: A comprehensive guide to methods and measures. Oxford University Press.

Horstmann, G., Herwig, A., \& Becker, S. I. (2016). Distractor dwelling, skipping, and revisiting determine target absent performance in difficult visual search. Frontiers in Psychology, 7, 1152. https:// doi.org/10.3389/fpsyg.2016.01152

In-Albon, T., Kossowsky, J., \& Schneider, S. (2010). Vigilance and avoidance of threat in the eye movements of children with separation anxiety disorder. Journal of Abnormal Child Psychology, 38(2), 225-235. https://doi.org/10.1007/s10802-009-9359-4

La Greca, A. M., \& Stone, W. L. (1993). Social anxiety scale for children-revised: Factor structure and concurrent validity. Journal of Clinical Child Psychology, 22(1), 17-27. https://doi.org/10.1207/ s15374424jccp2201_2

Lazarov, A., Abend, R., \& Bar-Haim, Y. (2016). Social anxiety is related to increased dwell time on socially threatening faces. Journal of Affective Disorders, 193, 282-288. https://doi.org/10. 1016/j.jad.2016.01.007

Liang, C. W., Tsai, J. L., \& Hsu, W. Y. (2017). Sustained visual attention for competing emotional stimuli in social anxiety: An eye tracking study. Journal of Behavior Therapy and Experimental Psychiatry, 54, 178-185.

Liversedge, S. P., Paterson, K. B., \& Pickering, M. J. (1998). Eye movements and measures of reading time. In G. M. Underwood (Ed.), Eye guidance in reading and scene perception. Elsevier.

Ludwig, C. J. H., Davies, J. R., \& Eckstein, M. P. (2014). Foveal analysis and peripheral selection during active visual sampling. Proceedings of the National Academy of Sciences of the United States of America, 111, E291-E299.

MacLeod, C., \& Clarke, P. J. (2015). The attentional bias modification approach to anxiety intervention. Clinical Psychological Science, 3(1), 58-78. https://doi.org/10.1177/2167702614560749

Mathews, A., \& Mackintosh, B. (1998). A cognitive model of selective processing in anxiety. Cognitive Therapy and Research, 22(6), $539-560$.

Melfsen, S., \& Florin, I. (1997). Ein Fragebogen zur Erfassung sozialer Angst bei Kindern (SASC-R-D). Kindheit Und Entwicklung, 6(4), 224-229.

Melfsen, S., Florin, I., \& Warnke, A. (2001). Sozialphobie und Angstinventar für Kinder (SPAIK). Hogrefe.

Mogg, K., \& Bradley, B. P. (1998). A cognitive-motivational analysis of anxiety. Behaviour Research and Therapy, 36(9), 809-848. https://doi.org/10.1016/S0005-7967\%2898\%2900063-1

Pelissolo, A., Abou Kassm, S., \& Delhay, L. (2019). Therapeutic strategies for social anxiety disorder: Where are we now? Expert Review of Neurotherapeutics, 19(12), 1179-1189. https://doi.org/ 10.1080/14737175.2019.1666713

Pergamin-Hight, L., Bitton, S., Pine, D. S., Fox, N. A., \& Bar-Haim, Y. (2016a). Attention and interpretation biases and attention control in youth with social anxiety disorder. Journal of Experimental 
Psychopathology, 7(3), 484-498. https://doi.org/10.5127/jep. 053115

Pergamin-Hight, L., Pine, D. S., Fox, N. A., \& Bar-Haim, Y. (2016b). Attention bias modification for youth with social anxiety disorder. Journal of Child Psychology and Psychiatry, 57(11), 1317-1325. https://doi.org/10.1111/jcpp.12599

Schmidtendorf, S., Wiedau, S., Asbrand, J., Tuschen-Caffier, B., \& Heinrichs, N. (2018). Attentional bias in children with social anxiety disorder. Cognitive Therapy and Research, 42(3), 273-288. https://doi.org/10.1007/s10608-017-9880-7

Schneider, S., Unnewehr, S., \& Margraf, J. (2009). Kinder-DIPS. Diagnostisches Interview bei psychischen Störungen im Kindes- und Jugendalter. Springer.

Schwarzer, G., Huber, S., \& Duemmler, T. (2005). Gaze behavior in analytical and holistic face processing. Memory and Cognition, 33, 344-354.

Seefeldt, W. L., Krämer, M., Tuschen-Caffier, B., \& Heinrichs, N. (2014). Hypervigilance and avoidance in visual attention in children with social phobia. Journal of Behavior Therapy and Experimental Psychiatry, 45(1) 105-112. https://doi.org/10.1016/j.jbtep. 2013.09.004
Shechner, T., Jarcho, J. M., Britton, J. C., Leibenluft, E., Pine, D. S., \& Nelson, E. E. (2013). Attention bias of anxious youth during extended exposure of emotional face pairs: An eye tracking study. Depression and Anxiety, 30, 14-21. https://doi.org/10.1002/da. 21986

Weierich, M. R., Treat, T. A., \& Hollingworth, A. (2008). Theories and measurement of visual attentional processing in anxiety. Cognition and Emotion, 22(6), 985-1018. https://doi.org/10.1080/02699 930701597601

Williams, J. M. G., Watts, F. N., MacLeod, C., \& Mathews, A. (1988). Cognitive psychology and emotional disorders. John Wiley and Sons.

Yoon, K. L., \& Zinbarg, R. E. (2008). Interpreting neutral faces as threatening is a default mode for socially anxious individuals. Journal of Abnormal Psychology, 117(3), 680.

Publisher's Note Springer Nature remains neutral with regard to jurisdictional claims in published maps and institutional affiliations. 